\title{
Anton Tchékhov chez les cannibales
}

Texte exotique et exotisme du texte dans La Sauterelle

\section{Boris Czerny}

\section{(2) OpenEdition}

\section{Journals}

Édition électronique

URL : http://journals.openedition.org/edl/386

DOI : $10.4000 /$ edl.386

ISSN : 2296-5084

Éditeur

Université de Lausanne

Édition imprimée

Date de publication : 15 septembre 2009

Pagination : 85-102

ISBN : 978-2-940331-20-8

ISSN : 0014-2026

Référence électronique

Boris Czerny, «Anton Tchékhov chez les cannibales », Études de lettres [En ligne], 2-3 | 2009, mis en ligne le 15 septembre 2012, consulté le 22 décembre 2020. URL : http://journals.openedition.org/edl/ 386 ; DOI : https://doi.org/10.4000/edl.386 


\section{ANTON TCHÉKHOV CHEZ LES CANNIBALES. TEXTE EXOTIQUE ET EXOTISME DU TEXTE DANS "LA SAUTERELLE"}

L'analyse d'une nouvelle de Tchékhov permet de dépister des références inattendues aux univers exotiques. Mieux, le récit lui-même intègre des mécanismes de représentations proches de ceux qui fonctionnent dans le genre du roman colonial.

Traiter de l'exotisme chez Anton Tchékhov semble relever de la gageure. En effet, à la fin du XIX ${ }^{e}$ siècle, à l'époque où la Russie entrait dans la modernité et où se développaient de nouveaux moyens de locomotion, l'univers de Tchékhov restait circonscrit aux limites d'une province russe figée dans le temps. Rien, en apparence, ne semble suggérer l'expression d'un exotisme qui serait défini comme un attrait particulier pour «ce qui est relatif [...] à un pays étranger, généralement lointain ou peu connu et qui a un caractère naturellement original dû à sa provenance» ${ }^{1}$. Pourtant, à la lecture de certaines œuvres de Tchékhov, le lecteur ressent parfois un dépaysement qui n'est pas provoqué par la distance dans le temps et l'espace entre la Russie de la fin du XIX et l'Europe du début du XXI e siècle. L'analyse de La Sauterelle permet d'expliciter cette impression. En effet, cette œuvre écrite en 1891 comporte, selon nous, de nombreux éléments relevant à la fois d'une esthétique exotique apparente - dans les décors et le paysage - et de la recherche d'une écriture "exotisante». Ces aspects de La Sauterelle en tant que texte exotique et relevant d'une stratégie exotique constitueront les deux volets de notre étude.

I. Trésor de la langue française: http://atilf.atilf.fr/tlf.htm, article "Exotique», consulté le 25/08/2008. 
Texte exotique

It is a suicide to be abroad. But what is it to be at home? A lingering dissolution.

Samuel Beckett, All the Fall

Dès sa première apparition dans la langue littéraire française, l'exotisme est lié à l'accomplissement d'un voyage. Ainsi, en 1552, avec l'édition définitive du Quart Livre, Rabelais introduit le terme "exotique", le glose aussitôt, selon un procédé familier à la langue française du $\mathrm{XVI}^{\mathrm{e}}$ siècle, par le terme plus compréhensible de "pérégrines» exposées sur le môle des docks de Medhamoti, l'île de «nulle part» qui est la première étape du voyage de Pantagruel et de Panurge à destination de l'oracle Babuc ${ }^{2}$. Cependant, si le déplacement vers un ailleurs riche de promesses conditionne la perception d'un dépaysement, les destinations suscitant les espoirs de fascination et de découvertes ont fortement varié selon les époques et les lieux de départ. Cette diversité rend bien relative la notion même d'exotisme. Néanmoins, il est possible de contourner la difficulté d'une définition absolue en déterminant les points communs de ces multiples expéditions du connu vers l'inconnu. Pour chacun de ces voyages, l'exotisme apparaît comme le produit d'une rencontre entre la représentation préconçue d'un lieu et la réalité quotidienne.

Dans le cas de Montaigne dans les Essais ou de Diderot dans le Supplément au voyage de Bougainville, l'exotisme va de pair avec la recherche du bonheur virginal dans l'âge d'or d'un état primitif, s'opposant à la perversion sociale et morale de la société contemporaine ${ }^{3}$. Parfois le résultat de la confrontation est décevant. Ainsi Medamothi, la première étape de la navigation de Pantagruel et de Panurge, est une île de l'illusion, un "palais des mirages " ${ }^{4}$. Aux XIX ${ }^{\mathrm{e}}$ et $\mathrm{XX}^{\mathrm{e}}$ siècles, l'exotisme s'exprima souvent sous la forme d'un rejet méprisant pour la

2. Pour le rapport entre exotisme et voyage voir, entre autres, V. Segalen, Essai sur l'exotisme. Une Esthétique du divers. Textes sur Gauguin et l'Océanie; J.-M. Moura, Lire l'exotisme; R. Célestin, From Canibals to Radicals. Figures and Limits of Exoticism; F. Lestringant, "L'Exotisme en France à la Renaissance de Rabelais à Léry"; J.-M. Moura, La Littérature des lointains. Histoire de l'exotisme européen au $20^{e}$ siècle.

3. J.-M. Blanchard, "Of Cannibalism and Autobiography», p. 676; J. Starobinski, Montaigne in Motion, p. 20.

4. V.-L. Saulnier, Rabelais II. Rabelais dans son enquête, p. 48; Traditions polémiques: Cahiers Verdun-Louis Saulnier, 2. 
culture - ou plutôt l'absence de culture - de l'Autre exotique. Ce type de rapport est caractéristique de la production littéraire coloniale dont la filiation avec la littérature dite exotique a fait l'objet de nombreuses études ${ }^{5}$. Ainsi, la supériorité de la culture blanche européenne est soulignée sans nuance chez le prolifique écrivain colonialiste Paul Vigné d'Octon, aujourd'hui peu connu, ou chez des auteurs plus célèbres comme Pierre Loti et Paul Morand dont certains écrits « exotiques» susciteraient l'indignation aujourd'hui.

Notre propos n'est pas de céder à un prêchi-prêcha moralisateur, mais de cerner les caractéristiques d'une littérature dite exotique.

Que le voyage soit motivé par des raisons apparemment nobles et honorables, ou par le désir d'affirmer une hégémonie culturelle et économique, l'exotisme se réalise à l'intérieur d'un espace discursif triangulaire dont les sommets sont représentés respectivement par l'espace d'arrivée, le voyageur et le lieu de départ que nous appellerons, de manière conventionnelle, "la maison». La distance entre ces trois points est par conséquent une donnée secondaire. Peu importe que le lieu visité soit proche ou lointain. Bien souvent en littérature il n'est pas indiqué, comme dans Robinson Crusoé, ou se confond avec d'autres endroits visités. Ainsi, Voltaire ne se souciait nullement de donner quelque couleur locale spécifique à la peuplade dont il parlait. De même, chez Flaubert ou chez Sade, les déplacements n'apprennent rien sur Angers, Naples, Astrakhan ou Paris. La variation de la surface de ce triangle «exotique» explique la diversité de perception d'un paysage ou d'une personne qui, par référence à la "maison», apparaît à André Gide comme étant, «la quintessence de l'exotisme», «d'apparence très exotique», «vraiment» ou "violemment exotique», ou, au contraire, "fort peu exotique» et "aussi peu exotique que possible» ${ }^{6}$.

Le constat de la relativité de l'exotisme ainsi que la mise en évidence du triple rapport entre le voyageur, le lieu visité et l'espace de départ comme donnée nécessaire à l'expression d'un sentiment de dépaysement

5. P. Jourda, L'Exotisme dans la littérature française de Chateaubriand; Exotisme et Création; J.-C. Brechet, Le Voyage en Orient; Y. Khibiehler et R. Goutalier, La Femme au temps des colonies; J. Yee, Clichés de la femme exotique. Un Regard sur la littérature coloniale française entre 1871 et 1914.

6. André Gide est un des auteurs contemporains les plus cités à la rubrique «Exotique» dans la base textuelle Frantext http://atilf.atilf.fr/frantext.htm que nous utilisons ici. 
exotique, permettent de relativiser l'absence apparente d'exotisme chez un auteur dont l'œuvre semble ancrée dans la seule réalité de son pays, comme c'est le cas de Tchékhov.

\section{«La Sauterelle»}

Le mot du titre se réfère à l'hérö̈ne de la nouvelle (il n'apparaît pas dans le texte) et renvoie à la célèbre fable de Krylov, reprise de «La Cigale et la Fourmi » de Jean de La Fontaine, où il qualifie la libellule, version russe de la cigale. Le sujet de la nouvelle est simple. Composée de huit chapitres, elle raconte quelques mois dans la vie d'Olga Ivanovna, après son mariage avec un médecin, Ossip Stepanovitch Dymov, dont elle a fait la connaissance au moment du décès de son père. Dymov est totalement dévoué à la pratique de la médecine et à la recherche scientifique. Elle, en revanche, mène une vie mondaine intense, entourée d'artistes et d'écrivains, à la recherche permanente de "gens extraordinaires». Elle néglige Dymov, trop commun, passe l'été à la campagne en compagnie d'un groupe d'amis, tombe amoureuse d'un peintre, un certain Riabovski. L'histoire d'amour tourne court. Au dernier chapitre, l'épouse volage rentre à la maison. Lorsque son mari meurt, parce qu'il a contracté la diphtérie en tentant de sauver un enfant, Olga comprend qu'elle n'a pas su reconnaître près d'elle l'homme extraordinaire qu'elle cherchait tant.

Le récit est structuré par deux lignes opposées, représentées respectivement par Olga et son mari. La jeune femme est constamment en mouvement, les déplacements de Dymov sont limités. Placée dans une perspective exotique, l'épouse joue le rôle de l'exploratrice. En effet, sa vie apparaît comme une succession de trajets, parfois à l'intérieur de sa propre maison, comme c'est le cas au début du deuxième chapitre; mais le plus souvent elle court d'une exposition de peinture à l'atelier de sa couturière, d'un spectacle au théâtre ou d'un concert à un souper chez des amis. Dans le chapitre IV, Olga effectue avec son amant un voyage sur la Volga. Le motif de l'éloignement de la «maison» est explicité. Sur le pont du bateau sur lequel ils voyagent, la jeune femme rêve de devenir peintre, du succès qui «l'attend quelque part dans le lointain, au-delà de cette nuit de pleine lune, dans un espace illimité». Exaltée, elle se laisse séduire. La vie avec son mari lui semble insipide: 
Elle voulait penser à son mari, mais tout son passé avec Dymov, avec les soirées à la maison, lui semblait étroit, insignifiant et fade, inutile et lointain, si lointain?

Cependant, après quelques semaines partagées avec Riabovski, Olga veut rentrer chez elle. L'attente du bateau qui va la ramener au port est comme un écho à Ulysse et à son espoir de revoir Ithaque où l'attend Pénélope. Olga ne supporte plus les difficiles conditions de son «expédition" sur la Volga. Les paroles du narrateur pourraient aisément trouver leur place dans quelque récit d'aventures lointaines:

[...] Elle se transporta en pensée dans le salon, dans le bureau de son mari, et elle s'imagina qu'elle était assise immobile près de Dymov et jouissait du calme et de la propreté des lieux et que le soir elle était au théâtre et qu'elle écoutait Mazzini. Et la tristesse d'être si loin de la civilisation, du bruit de la ville et de ses amis provoqua une légère douleur dans sa poitrine ${ }^{8}$.

Pourtant, revenue à la maison, au chapitre VII, Olga ressent à nouveau des envies de départ. La destination choisie, la Crimée, symbolise un exotisme proche, un espace baigné de soleil où la vie est douce et la végétation généreuse. Mais la mort soudaine de son mari rend ce rêve de voyage impossible. C'est d'ailleurs une des principales caractéristiques des déplacements d'Olga et de son mari. Ces deux voyageurs semblent frappés par une malédiction. Dymov, à l'issue de l'unique voyage qui le conduit à la maison de campagne où son épouse passe l'été, se voit refuser l'accès de la datcha. Plus exactement, sa femme ne lui permet pas de rester. De même, quand, au chapitre VII, la «Sauterelle» revient chez elle, elle n'est pas autorisée à entrer dans la chambre de son mari qui est grièvement malade. Les trajets dans l'espace, signalés par des commentaires lapidaires qui évoquent le style des journaux de voyage, apparaissent comme des cercles inachevés. Les personnages sont condamnés à l'errance.

7. A. P. Čehov, Poprygun'ja, p. 305-306. Dorénavant nous indiquerons pour les citations de La Sauterelle uniquement le numéro de la page et du chapitre. Sauf indication contraire, les traductions sont de nous.

8. Chap. V, p. 309. 
Olga décore sa maison avec des objets qui suggèrent ces errances. Le début du chapitre II contient une description de l'aménagement intérieur du salon:

Olga Ivanovna avait entièrement recouvert les murs du salon d'études, les siennes et celles d'autres peintres, certaines étaient encadrées, d'autres non, et à côté du piano et d'un meuble elle avait aménagé un petit endroit d'une douce intimité où s'accumulaient des ombrelles chinoises, des chevalets, des chiffons multicolores, des poignards, des bustes et des photographies. Dans la salle à manger, elle avait tapissé les murs d'images d'Epinal, elle avait accroché des chaussures de tille et des serpes, dans un coin elle avait mis une faux et un râteau, ce qui donnait à l'ensemble un aspect russe. Afin que la chambre ressemble à une grotte, elle avait drapé le sol et le plafond d'un tissu de couleur sombre et avait suspendu au-dessus du lit une lanterne vénitienne, et près des portes elle avait disposé une figurine avec une hallebarde 9 .

Les objets disparates mis ensemble s'enrichissent de cette qualité «exotique» dont ils étaient dépourvus au départ. Ils sont transposés dans une nouvelle réalité et peuvent être exhibés comme la peau d'une bête sauvage ou comme des arbres et des fleurs exotiques transplantés dans le jardin de Tartarin:

O le jardin de Tartarin, il n'y en avait pas deux comme celui-là en Europe. Pas un arbre du pays, pas une fleur de France; rien que des plantes exotiques, des gommiers, des calebassiers, des cotonniers, des manguiers, des bananiers, des palmiers, un baobab, des nopals, des cactus, des figuiers de Barbarie, à se croire en pleine Afrique centrale à dix mille lieues de Tarascon ${ }^{10}$.

Leur disposition est destinée à produire un effet d'accumulation. A défaut d'être définie avec fermeté, la valeur de l'exotisme peut être approchée grâce au procédé de l'énumération. Dans les deux cas, celui de Tartarin et celui de la "Sauterelle", les descriptions fonctionnent comme des cartes postales. Elles permettent de rendre accessible la réalité extérieure. Ce faisant elle favorise l'aporie de l'exotisme en le codifiant. Lobjet exotique devient conventionnel et banal. Il acquiert le

9. Chap. II, p. 301 (souligné par nous).

Io. A. Daudet, Tartarin de Tarascon, p. 2. 
statut d'un signal de reconnaissance rapide et sans ambiguïté d'une altérité maîtrisée, vidée de sa vitalité. La faux et la lanterne vénitienne de la "Sauterelle», le calebassier chez Tartarin, ne sont pas destinés à donner un effet de réel. Ils créent un condensé d'exotisme qualifié de "sinité" par Roland Barthes au sujet de la "pipe» et l'«opium» pour la Chine ${ }^{11}$. D’autres détails, semés dans le récit de Tchékhov, fonctionnent comme des éléments métonymiques d'un attirail exotique où la «Sauterelle»va puiser afin de donner une illusion d'originalité. C'est notamment le cas du visage du docteur qu'elle voit ressembler à l'ours, au tigre du Bengale, enfin à un «bédouin".

Les exemples cités montrent que l'exotisme contient en lui une déception potentielle. L'effacement dans la ressemblance menace l'objet, les personnes ou les situations considérés, un instant, comme exotiques. Ce processus d'usure explique le mouvement permanent qui anime Olga, obsédée par la crainte de devenir banale aux yeux de ses amis et de son amant. Elle recherche l'illusion dont elle est elle-même victime. Elle collectionne les objets et multiplie les visites chez des personnes qui sont plus extraordinaires les unes que les autres, au risque d'une régression qui la condamne à perdre l'amour du peintre. L'hérö̈ne ne possède aucun talent artistique et le peintre amoureux se révèle être un gandin infatué. Les masques tombent. L'exotisme apparaît comme le vernis superficiel et trompeur d'une banalité déguisée. Le jeu et le travestissement occupent une place importante dans l'œuvre de Tchékhov et dans le récit que nous étudions. Ainsi que nous l'avons indiqué précédemment, Dymov se transforme en bédouin. Parmi les connaissances de la jeune femme figurent de nombreux acteurs. Le début du chapitre II est consacré aux spectacles amateurs que donne la jeune femme et à ses relations amicales avec une actrice. Le théâtre (ce mot apparaît dans le récit au moins cinq fois, dans différents contextes) est une des sorties privilégiées d'Olga. Celle-ci devient le support de sa propre représentation exotique: elle se voit, tache multicolore, à la manière des "expressionnistes français» (chapitre III).

L'érotisme que sous-tend l'évocation d'une nature nue (chapitre V) est une donnée essentielle du récit, mise en évidence dans le sujet même. Certaines scènes, comme celle du baiser sur les lèvres échangé entre les deux amants sur le pont du bateau (chapitre II), se caractérisent par leur

II. R. Barthes, «Le Mythe aujourd'hui», p. 692. 
tonalité convenue. Le cadre même, le bateau, ainsi que l'opposition entre l'artiste et le docteur, la création artistique et la science, sont des situations d'une rare banalité que l'on retrouve en particulier dans la littérature coloniale de la fin du XIX ${ }^{\mathrm{e}}$ siècle et du début du $\mathrm{XX}^{\mathrm{e}}$ siècle ${ }^{12}$. Les exposés des fantasmes sexuels des colons européens en Afrique ou en Indochine sont bien souvent des fadaises, d'un conformisme affligeant ${ }^{13}$. Ils associent deux motifs majeurs. Le premier est celui du caractère purement physiologique des rapports sexuels entre l'homme européen et la femme "exotique». Faire l'amour à une femme africaine est une reproduction individualisée de l'action civilisatrice de la France sur les "tribus de sauvages». Le second thème renvoie à l'image de la femme fatale dont l'avènement littéraire correspond doublement à la crise du roman dans la littérature européenne et, sur un autre plan, à l'essor du colonialisme ${ }^{14}$. Cette femme fatale est généralement originaire de l'Orient. Souvent, elle est juive ${ }^{15}$. Cependant, la géographie a peu d'importance. L'essentiel est là encore le signe qui marque une rupture avec le quotidien de la «métropole».

Olga quitte la maison - l'espace d'origine - pour connaitre l'amour. La situation est opposée à celle que l'on rencontre dans la majorité des romans exotico-colonialistes où la femme est rarement la représentante de la puissance dominante. Dans le récit de Tchékhov, la «Sauterelle» soumet les hommes à sa volonté et à ses désirs. Dès les premières lignes du texte elle apparaît entourée d'une foule d'admirateurs. Plus loin, lors du séjour à la campagne, elle est la seule femme parmi de nombreux hommes. Enfin, sa domination sur la gent masculine se concrétise par la scène du baiser sur le pont du bateau. La conduite d'Olga rappelle celle des femmes fatales. Elle renvoie également au fantasme sexuel du «sérail» ou du «harem» tel qu'il était illustré dans la littérature coloniale. Cette représentation «idéalisée» de l'accomplissement du désir sexuel ne dépend pas, ou peu, de la localisation exotique et lointaine, mais de la multiplicité des personnes (ici des hommes) dépendantes du

I2. J. Yee, Clichés de la femme exotique. Un Regard sur la littérature coloniale française entre 1871 et 1914.

I3. R. Girardet, L'Idée coloniale en France; A. Ruscio, Amours coloniales, aventures et fantasmes exotiques de Claire de Duras à Georges Simenon, p. 10.

I4. M. Dottin-Orsini, Cette femme qu'ils disent fatale.

15. L. A. Klein, Portrait de la juive dans la littérature française. 
bon vouloir du maître ${ }^{16}$. Le comportement de la «Sauterelle», réprouvé par son entourage, est une manière d'exprimer ses distances avec le mode de vie auquel la contraint la morale commune.

La sexualité dans un ailleurs exotique s'inscrivant dans un espace qui n'est pas uniquement géographique ${ }^{17}$, fut un des moyens les plus fréquemment utilisés pour justifier le colonialisme ou, au contraire, pour questionner la validité du "centre» ou de la maison selon notre terminologie. Cette double attitude apparaît nettement à la comparaison du texte de Bougainville et de l'essai de Diderot intitulé Supplément au voyage de Bougainville. L'explorateur est un observateur neutre. Il reste un émissaire de la "maison" et, de façon très révélatrice, voyage sur un navire dont le nom, France, évoque l'attachement au centre. Bougainville se contente de noter la présence des corps nus de femmes. Il banalise l'exotisme. Diderot, qui n'effectue pas le voyage, se réfère à la nudité et à la sexualité des Tahitiens pour critiquer le caractère artificiel des sociétés européennes. La vie sexuelle des «sauvages» est plus naturelle que celle des sociétés dites civilisées.

Dans La Sauterelle, la remise en question de la norme est relative. Tout rentre dans l'ordre. La femme retrouve la position d'être soumis au bon vouloir du maitre. Lorsque l'hérö̈ne rend visite à son ancien amant, il l'éconduit: d'autres femmes ont pris sa place.

Mais le retour à une situation banale ne limite pas pour autant la portée exotique du texte. Ainsi, Dymov est à la fois un sauvage soumis par son épouse à un examen morphologique ("Vous ne voyez pas qu'il y a en lui quelque chose qui évoque l'ours? Actuellement son visage est de trois quarts et mal éclairé, mais quand il se tournera vers nous, regardezdonc son front...») et le représentant symbolique d'un lieu de départ qui constitue un des angles de la relation "exotique» formée du par la «maison ", le voyageur et l'espace exploré ${ }^{18}$. Le statut symbolique de Dymov est affirmé dès le début du récit. Olga fait sa connaissance lors du décès de son père qui exerçait également le métier de médecin. Dymov qui va mourir à la fin du récit apparaît comme un double du père de sa future

16. A. Ruscio, Amours coloniales, aventures et fantasmes exotiques de Claire de Duras à Georges Simenon, p. 15.

17. P. Ricœur, Philosophie de la Volonté, p. 94.

I8. La nature de cette relation est exposée par Roger Célestin, From Canibals to Radicals. 
femme. Cette "parenté» est renforcée par la façon qu'a Dymov d'appeler sa femme "maman». Enfin, le nom même de Dymov est pratiquement toujours associé au mot «maison». Dans ce contexte, le vocable «frère» employé par Dymov pour désigner son ami Korostelev peut sembler ambigu. Il peut être une simple forme d'adresse ou l'expression d'un lien familial caché qui renforce le statut de la "maison» en tant qu'espace de référence. Les voyages d'Olga seraient donc bien des échappées hors d'un espace connu vers des destinations plus ou moins lointaines d'où elle ramène des trophées exotiques. Le sujet du récit peut être résumé de cette façon.

\section{Exotisme du texte}

La distanciation tchékhovienne a fait l'objet d'un nombre certain d'études axées principalement sur l'éthique de l'écrivain. Dès 1893, le journaliste et poète Piotr Pertsov fustigea l'indifférence de Tchékhov pour les grandes questions sociales et humaines de son époque. Cet éloignement de la réalité environnante fut mis au compte de l'ironie méprisante de l'écrivain ${ }^{19}$. Saveli Senderovich, qui prête une attention particulière aux répétitions et aux allusions métonymiques, voit l'ironie tchékhovienne comme un procédé littéraire résultant de l'enchâssement de plusieurs textes les uns dans les autres.

L'idée de l'imbrication de plusieurs récits les uns dans les autres semble paradoxale. Pour reprendre une appréciation généralement admise, "rien ne se passe» dans La Sauterelle. Le rythme est donné par les multiples répétitions et dédoublements. Ainsi Dymov et Riabovski apparaissent-ils comme des doubles imparfaits qui incarneraient cette opposition classique et "rousseauiste» ${ }^{20}$ : la vérité contre l'art et la rhétorique, l'être contre le paraître, le propre contre le figuré, la nature sauvage et exotique contre la civilisation. Une telle distinction entre les deux personnages est justifiée. Il est cependant possible de noter que le médecin et l'artiste possèdent des points communs. Ainsi, au tout début du récit, il est précisé que le docteur travaille beaucoup et gagne un salaire insignifiant de cinq cents roubles. Ce même chiffre apparaît

19. P. Percov, «Iz' jany tvorčestva», p. 80-93, 180-216, 271.

20. D. Leduc-Fayette, Jean-Jacques Rousseau et le mythe de l'Antiquité, p. 71-101. 
quelques lignes plus loin pour indiquer le prix d'une peinture vendue par Riabovski. L'«amour" pour Olga également rapproche les deux hommes. Cette interchangeabilité semble être facilitée par la nature même des personnages. Aussi, Dymov est-il un conglomérat de traits opposés qui s’annulent:

[...] Dans cette joyeuse bande, Dymov semblait être un étranger, une personne en trop et petite, alors qu'il était de grande stature et large d'épaules. Il donnait l'impression d'avoir sur le dos un habit qui n'était pas le sien et de porter une barbe semblable à celles des commis. En fait, $s$ 'il avait été un écrivain ou un artiste, on aurait dit que sa barbe le faisait ressembler à Zola ${ }^{21}$.

Le médecin est grand et petit à la fois. Sa barbe est un peu ridicule, mais peut devenir un attribut noble, rapprochant le héros de l'intelligentsia française. Ces paradoxes nous amènent à soupçonner que Dymov n'existe pas en tant que personnage «réel». Il est, comme le suggère la première syllabe de son nom en russe, de la fumée: Dym-ov. Cette lecture est corroborée par sa ressemblance avec l'écrivain français: Zola signifie en russe la cendre. Dymov, comme la plupart des personnages présents dans La Sauterelle, se distingue également par une formidable capacité à s'incarner sous de multiples avatars, tout en restant vide, sans apparence précise. Au début, il est un mari, un homme ordinaire, un médecin et conseiller honoraire, un interne et dissecteur. Son nom est pour les invités d'Olga aussi peu important que celui d'un quelconque Sidorov ou Tarassov. Ensuite, il prend les apparences d'un homme intelligent et noble, d'un maitre d'hôtel, d'un ours, d'un tigre du Bengale et du père d'Olga. Dans le chapitre IV, il disparaît et son épouse doute de son existence réelle.

Cette capacité de Dymov et des autres personnages à prendre différents aspects fait écho à la passion d'Olga pour les costumes et la couture. Cette occupation souligne sa futilité. Elle illustre également la structure même du récit. Ce dernier est un assemblage de mots ou d'expressions détournés de leur emploi premier, inversés ou retournés comme des vieilles vestes. La première allusion à la couture est donnée au début du chapitre II. Une simple phrase comportant des énumérations donne la tonalité générale du récit:

2I. Chap. I, p. 299. 
Très souvent, à partir d'une vieille robe retaillée, et de morceaux sans valeur de tulle, de dentelles, de pluche et de soie, elle faisait des miracles, quelque chose de charmant, non pas une robe, mais un rêve ${ }^{22}$.

Les mots "miracles", "charmant", "rêve» font basculer le texte dans un autre univers fictionnel, dans le monde des fées qui transforment les choses et les êtres d'un coup de baguette magique. Par la suite, la mutation du récit en conte, ou, plus exactement, en parodie de conte, est progressive. Elle ne produit donc aucun effet de surprise. Une nouvelle version du récit est ébauchée au moment de l'arrivée de Dymov à la datcha au chapitre III. A nouveau, comme sur le bateau ou lors des séances de couture, la scène se passe à la limite du rêve et de la réalité. A son mari qui vient de se présenter, Olga dit qu'elle a rêvé de lui toute la nuit. A partir de cet instant, le récit réunit les différents attributs traditionnels des contes. Le rôle de la princesse est tenu par la «Sauterelle». Le prince est incarné par Dymov que son épouse surnomme son "sauveur». Bien évidemment, dans le contexte d'une imitation du genre fantastique, la quête est insignifiante, même si elle est conforme au code du genre. L'objet que doit aller quérir le héros est un motif traditionnel des récits de princesses. Il s'agit d'une robe rose, de gants et de morceaux de tulle. Dans certains contes, comme Cendrillon, les limites temporaires sont fixées précisément ce qui permet d'intensifier le suspense, car après une certaine heure, les charmes cessent d'agir ${ }^{23}$. Les carrosses redeviennent citrouilles et les princesses des souillons. On peut donc supposer que si Dymov ne remplit pas sa mission à temps, un malheur arrivera par la faute de l'adversaire du docteur, le peintre Riabovski. En effet, l'artiste possède le don de séduction et de reproduction du réel. Il détourne donc la princesse vers le monde des apparences. Pour mieux tromper la vigilance de sa victime, il n'hésite pas à inverser les rôles et à déclarer que la jeune femme est "envoûtante» ${ }^{24}$.

Cette dernière question est étrange. A moins de considérer qu'Olga est une fée. Elle exerce un charme, au sens propre du terme, et Riabovski se méfie d'elle et de ses pouvoirs. Ainsi, il se saisit «subitement " ${ }^{25}$ d'un

22. Chap. II, p. 300.

23. Les chapitres I-IV de La Sauterelle comportent de nombreuses indications se rapportant aux heures «fatales» de midi et minuit.

24. Poprygun'ja, chap. IV, p. 306.

25. Ibid., chap. IV, p. 308. 
couteau et lacère un dessin: un comportement incompréhensible à moins de supposer que la peinture inspirée par Olga exerce un maléfice. Dans la scène à la datcha, en chargeant son mari de lui rapporter sa robe rose, la jeune femme lui dit de se dépêcher, car "elle n'avait rien à se mettre, au sens propre». Or si, au sens propre, la jeune femme n'a rien à se mettre, elle est nue, telle une sorcière.

Un écho à cette nudité est donné par la description du paysage faite juste après la scène de la lacération du tableau par Dymov.

Et on avait l'impression que la nature avait ôté de la Volga les luxueux tapis couvrant ses rives, les reflets de diamants des rayons, la transparence bleue de l'horizon, que tout ce qui donnait au paysage son élégance et son air pavoisé, avait été remisé dans des malles jusqu'au printemps suivant et les corneilles passaient en volant près de la Volga et se moquaient d'elle: «Tu es toute nue! Tu es toute nue!» 26

Olga est apparemment la créatrice de ce paysage animé. En effet, au chapitre II, la jeune femme a réalisé un croquis qu'elle montre à son amant. Cette esquisse préfigure la "peinture» de la Volga au chapitre V. Riabovski tient le tableau d'Olga dans ses mains et dit:

Voyons... Ce petit nuage-là jure: il n'est pas éclairé comme un nuage le soir. Le premier plan est, disons, trop mâché, et il y a quelque chose qui n'est pas vraiment juste... Et cette petite isba, on dirait qu'elle a avalé de travers et quelle émet un piaulement plaintif... ${ }^{27}$

On reconnaît dans ce dessin des éléments du paysage évoqué plus tard, tout comme la maison, où une vieille servante (autre image de sorcière) prépare le repas et où Olga se dispute avec Riabovski. La querelle annonce le départ d'Olga. Le peintre se demande comment elle compte partir: "A cheval sur un balai magique» ${ }^{28}$ ?

L'enchâssement des textes - d'un conte fantastique à l'intérieur d'un récit de facture classique contenant des éléments relevant éventuellement de la littérature coloniale - s'inscrit dans le projet poétique de l'auteur qui, à l'instar des peintres et artistes de son récit, se livre à la

26. Ibid., chap. V, p. 308.

27. Ibid., chap. II, p. 303.

28. Ibid. 
composition d'une œuvre «à la manière de... ». L'identité de l'œuvre originale "copiée» est donnée par le titre même. Une autre piste nous est soufflée par l'auteur lui-même, par l'entremise de la "Sauterelle». La jeune femme explique qu'elle n'aime pas le prénom de son mari qui rappelle un personnage de Gogol. La Sauterelle rappelle en effet la thématique et la poétique gogolienne. L'histoire d'un "sorcier» causant la perte d'un peintre rappelle Le Portrait. Le motif de la couture et du déguisement renvoie au Manteau. Mais c'est La Perspective Nevski qui semble être le vrai hypotexte de La Sauterelle. Chez Gogol, deux hommes qui se ressemblent et s'opposent en même temps suivent deux femmes dans la rue. Le premier, Piskarev, est peintre comme Riabovski. Son nom évoque en russe le bruit émis par un insecte. Il a donc un lien de parenté avec la "cigale-sauterelle» de Tchékhov. Il poursuit une femme brune, vision de la pureté, qui se révèle une prostituée. Le second personnage, un officier du nom de Pirogov, va essayer de séduire la blonde épouse d'un ferblantier allemand nommé Schiller. L'officier dont le nom (pirog signifie "tourte») traduit la nature grossièrement matérialiste, commandera au mari une gaine pour son poignard. Le nom de cet attribut militaire fait écho à la série d'objets qui marquent le décor de la rue où Piskarev suit la prostituée: le jeune héros romantique a l'impression que des hallebardes et des paires de ciseaux volent dans sa direction.

La présence des ciseaux, hallebardes, réverbères, illuminations, établit une passerelle entre La Sauterelle et La Perspective Nevski, ce dernier récit orientant le fonctionnement intérieur de la nouvelle de Tchékhov. Le motif «cannibale» les rapproche encore plus. A la fin du récit de Gogol, Pirogov se livre à un acte autophage en mangeant une tourte, une part de lui-même. Dans La Sauterelle, quand Olga rentre chez elle après son escapade sur la Volga, elle trouve son mari assis à table en train de manger une gelinotte, en russe riabtchik. Une seule page contient quatre occurences du morphème riab: dans le nom du peintre, dans le surnom affectif que lui donne Olga (Riaboucha), dans le nom de gelinotte qui, du coup, se confond avec celui de l'amant. "Mange la gelinotte», dit Dymov à Olga. Le récit d'une jeune femme trompant son ennui dans une relation éphémère avec un amant médiocre culmine en une scène de cannibalisme.

Le choix d'une histoire d'amour dépourvue de romantisme et d'une forme de narration concise, le récit et/ou la nouvelle, sont l'expression de la crise du roman. Tchékhov résout cette situation de crise en 
détournant les attributs habituels du roman, en particulier de la littérature coloniale. La situation finale élève le langage au rang d'élément supérieur de la transgression de la "centralité littéraire». L'écrivain russe réalise en l'occurrence ce que Flaubert considérait comme l'apogée, un «livre sur rien". Ainsi que le suggère Jean-Paul Sartre dans L'Idiot de la famille, dans le projet flaubertien, le désir de représenter un lieu exotique ou une femme, est entièrement placé sous le signe de l'impossible ${ }^{29}$. Chez Tchékhov, comme chez Flaubert, le véritable espace exotique est littéraire. Le voyage vers cet espace sauvage nécessite la remise en question des représentations banalisées; la scène "cannibale" de Tchékhov illustre bien cette attitude. Délesté des conventions, l'écrivain et le lecteur se trouvent devant un espace vide, l'espace de l'imagination qui est aussi celui de l'exotisme.

Boris Czerny

Caen

29. J.-P. Sartre, L'Idiot de la famille, p. 313. 


\section{BIBLIOGRAPHIE}

Barthes, R., "Le Mythe aujourd'hui", in Euvres complètes, I, Paris, Seuil, 1993.

Blanchard, J.-M., "Of Cannibalism and Autobiography", Modern Languages Notes, 93/4 (May 1978), p. 654-676.

Brechet, J.-C. (éd.), Le Voyage en Orient, Paris, Robert Laffont, 1985.

ČEHov, A. P., "Poprygun'ja», Izbrannoe, Moskva, Eksmo, 2003.

Célestin, R., From Canibals to Radicals. Figures and Limits of Exoticism,

Minneapolis, University of Minnesota Press, 1995.

Daudet, A., Tartarin de Tarascon, Paris, Dentu, 1872.

Dottin-Orsini, M., Cette femme qu'ils disent fatale, Paris, Grasset, 1993.

Exotisme et Création, Actes du Colloque, Lyon, L'Hermès, 1985.

Girardet, R., L'Idée coloniale en France, Paris, La Table ronde, 1972.

Jourda, P., L'Exotisme dans la littérature française de Chateaubriand, 1, Le Romantisme, Paris, Boivin, 1938.

Khibiehler, Y. et Goutalier, R., La Femme au temps des colonies, Paris, Stock, 1985.

Klein, L. A., Portrait de la juive dans la littérature française, Paris, Nizet, 1970.

Leduc-Fayette, D., Jean-Jacques Rousseau et le mythe de l'Antiquité, Paris, J. Vrin, 1974.

Lestringant, F., "L'Exotisme en France à la Renaissance de Rabelais à Léry", in Littérature et Exotisme, XVI et XVIIIe siècles, Dominique de Courcelles (éd.),Paris, Ecole des Chartes, 1997, p. 5-16.

Moura, J.-M., Lire l'exotisme, Paris, Dunod, 1992.

—, La Littérature des lointains. Histoire de l'exotisme européen au $20^{e}$ siècle, Paris, Honoré Champion, 1998.

Percov, P., "Iz'jany tvorčestva», Russkoe bogtastvo, 11 (1983), cité et reproduit in Čehov, Pro et Contra, Tvorčestvo A. P. Čehova v 
russkoj mysli konca 19-načala 20 veka (1887-1914), Igor Suhih

(éd.), Sankt-Peterburg, izd. Russ. Hristian. gumanit. inst., 2002.

Ricceur, P., Philosophie de la Volonté, I, Paris, Aubier, 1949.

Ruscio, A. (éd.), Amours coloniales, aventures et fantasmes exotiques de Claire de Duras à Georges Simenon, Bruxelles, Complexe, 1996.

SARTre, J.-P., L'Idiot de la famille (The Family Idiot), tr. Carole Cosman, Chicago, Chicago Univ. Press, 1993.

Saulnier, V.-L., Rabelais II. Rabelais dans son enquête, Paris, SEDESCDU, 1982.

Starobinski, J., Montaigne in Motion, (tr. A. Goldhammer), Chicago, Chicago Univ. Press, 1985.

Segalen, V., Essai sur l'exotisme. Une esthétique du divers. Textes sur Gauguin et l'Océanie (1917), Paris, Livre de Poche, 2004.

Traditions polémiques: Cahiers Verdun-Louis Saulnier, 2, Collection de l'Ecole Normale Supérieure de jeunes filles, 27, 1985.

Trésor de la langue française: http://atilf.atilf.fr/tlf.htm, article "Exotique» consulté le 25/08/2008.

YEE, J., Clichés de la femme exotique. Un Regard sur la littérature coloniale française entre 1871 et 1914, Paris, L'Harmattan, 2000. 
